\title{
A NOTE ON $S$-SPACES
}

E. G. BEGLE

An $S$-space is a normal topological space in which each covering by open sets has a refinement which is star-finite, that is, each set of the refinement meets only a finite number of sets of the refinement. Thus a compact (=bicompact) space is an $S$-space, and an $S$-space is paracompact [1]. ${ }^{1}$

In this note we discuss cartesian products in which one of the factors is an $S$-space. We show that if the other factor is compact, then the product is an $S$-space, and the dimension of the product does not exceed the sum of the dimensions of the factors. However, if both factors are $S$-spaces, the product need not be an $S$-space.

Theorem. Let $A$ be an n-dimensional $S$-space and $B$ an m-dimensional compact space. Then $A \times B$ is an $S$-space and $\operatorname{dim}(A \times B) \leqq n+m$.

By the dimension of a space we mean, of course, the Lebesgue dimension (cf. [2, p. 206]).

Let $\mathfrak{B}_{0}$ be an arbitrary covering of $A \times B$. We are going to construct a locally-finite cell complex, $D$, with $\operatorname{dim} D \leqq n+m$, a mapping $f$ of $A \times B$ onto $D$, and a covering $\mathfrak{Y}$ of $D$ such that $f^{-1}(\mathfrak{Y})$ is a refinement of $\mathfrak{W}_{0}$.

Let $a$ be any point of $A$. Each point of $a \times B$ is contained in an open set of the form $U \times V, U$ open in $A, V$ open in $B$, such that $U \times V$ is contained in an open set of $\mathfrak{B}_{0}$. For a fixed point $a \in A$, the set of all such $V$ 's is a covering of $B$, and hence a finite number of them, say $V_{a, 1}, V_{a, 2}, \cdots, V_{a, k(a)}$, form a covering $\mathfrak{B}_{a}$ of $B$. Let $U_{a}$ be the intersection of the corresponding $U$ 's.

The collection of all such sets $U_{a}$ is a covering of $A$. Hence there is a star-finite refinement $\mathfrak{U}$ of this covering whose order is no more than $n+1$. We may also assume $[2$, p. 210] that $\mathfrak{U}$ is normal, that is, that there is a mapping $\phi$ of $A$ onto $N(\mathfrak{U})$ such that each open set of $\mathfrak{U}$ is the inverse image, under $\phi$, of the star of a vertex of $N(\mathfrak{U})$.

We form a covering $\mathfrak{W}$ of $A \times B$ as follows: each set $U$ of $\mathfrak{U}$ is contained in some $U_{a}$, and with each $U_{a}$ is associated a covering $\mathfrak{B}_{a}$ of $B$. Form the product of $U$ with each set of $\mathfrak{B}_{a}$. The totality of these products forms $\mathfrak{W}$, and by construction, $\mathfrak{W}$ is a refinement of $\mathfrak{W}_{0}$.

Let $\theta$ be the mapping of $A \times B$ onto $N(\mathfrak{U}) \times B$ defined by $\theta(a \times b)$ $=\phi(a) \times b$, where $\phi$ is the above mapping of $A$ onto $N(\mathfrak{U})$. Each ele-

Received by the editors May 15, 1948.

${ }^{1}$ Numbers in brackets refer to the references cited at the end of the paper. 
ment of $\mathfrak{B}$ is thus mapped by $\theta$ onto an open set of $N(\mathfrak{U}) \times B$, so $\mathfrak{X}=\theta(\mathfrak{B})$ is a covering of $N(\mathfrak{H}) \times B$.

Now let $u_{1}$ be a fixed vertex of $N(\mathfrak{u})$ and let $S_{1}$ be the closed star of $u_{1}$, and, inductively, let $S_{i}$ be the closed star of $S_{i-1}$. Let $T_{1}=S_{1}$ and let $T_{i}, i>1$, be the closure of $S_{i}-S_{i-1}$, and let $R_{i}=T_{i-1} \cap T_{i}$.

Now $\bigcup_{i=1}^{\infty} S_{i}$ is a connected set which is both open and closed in $N(\mathfrak{U})$ and hence is a component of $N(\mathfrak{U})$. Since the constructions we make below can be made independently in each component, we may assume without loss of generality that $\bigcup_{i=1}^{\infty} S_{i}=N(\mathfrak{U})$.

Each vertex $u$ of $N(\mathfrak{H})$ corresponds to an open set $U$ of $\mathfrak{u}$ and, as above, each $U$ is contained in a set $U_{a}$ to which there corresponds a covering $\mathfrak{B}_{a}$ of $B$. Let $\mathfrak{B}_{1}^{\prime}$ be a finite covering of $B$ which is a common refinement of each $\mathfrak{B}_{a}$ which corresponds to a vertex of $T_{1}$. Let $\mathfrak{B}_{1}$ be a normal finite covering of $B$ which is of order not greater than $m+1$ and which is a star-refinement of $\mathfrak{B}_{1}^{\prime}$, that is, each set consisting of an element $\mathfrak{B}_{1}$ together with all the elements of $\mathfrak{B}_{1}$ which meet it is in an element of $\mathfrak{B}_{1}^{\prime}$.

In general, having obtained $\mathfrak{B}_{i-1}$, we obtain $\mathfrak{B}_{i}$ as follows: let $\mathfrak{B}_{i}^{\prime}$ be a common finite refinement of $\mathfrak{B}_{i-1}$ and of each $\mathfrak{B}_{a}$ which corresponds to a vertex of $T_{i}$. Let $\mathfrak{B}_{i}$ be a normal finite covering of $B$, of order not greater than $m+1$, which is a star-refinement of $\mathfrak{B}_{i}^{\prime}$.

Let $C_{i}$ be the finite cell-complex $T_{i} \times N\left(\mathfrak{B}_{i}\right)$. Since $\mathfrak{B}_{i}$ is a refinement of $\mathfrak{B}_{i-1}$, there is a projection $\pi_{i}$, a simplicial mapping, of $N\left(\mathfrak{B}_{i}\right)$ into $N\left(\mathfrak{B}_{i-1}\right)$. For each $i$, identify the subcomplex $R_{i} \times N\left(\mathfrak{B}_{i}\right)$ of $T_{i} \times N\left(\mathfrak{B}_{i}\right)$ with the subcomplex $R_{i} \times \pi_{i} N\left(\mathfrak{B}_{i}\right)$ of $T_{i-1} \times N\left(\mathfrak{B}_{i-1}\right)$. The result of these identifications is the cell-complex $D$. Since $N(\mathfrak{U})$ is at most $n$-dimensional, and $N\left(\mathfrak{B}_{i}\right)$, for each $i$, is at most $m$-dimensional, the highest possible dimension for a cell of $D$ is $n+m$.

Since each $\mathfrak{B}_{i}$ is normal, there is a corresponding mapping $\zeta_{i}$ of $B$ onto $N\left(\mathfrak{B}_{i}\right)$. Let $\zeta$ be the transformation of $N(\mathfrak{U}) \times B$ onto $D$ defined by setting $\zeta(p \times b)=p \times \zeta_{i}(b)$ for $p \in T_{i}-R_{i}$. Since each $\pi_{i}$ is continuous, so is $\zeta$. Now $f=\zeta \theta$ is a mapping of $A \times B$ onto $D$.

To construct the covering $\mathfrak{Y}$ of $D$, let $u$ be any vertex of $N(\mathfrak{u})$. Then $u$ is in some $R_{i}$. Let $v$ be a vertex of $N\left(\mathfrak{U}_{i-1}\right)$, and consider $u \times v$ as a vertex of $C_{i-1}$. Let $K$ be the star, in $C_{i-1}$, of $u \times v$. Then consider $u$ as a vertex of $T_{i}$, and let $v_{1}, v_{2}, \cdots, v_{s}$ be all the vertices of $N\left(\mathfrak{U}_{i}\right)$ which are mapped onto $v$ by $\pi_{i}$. Let $L$ be the union of the stars of $u \times v_{1}, \cdots, u \times v_{s}$ in $C_{i}$. Then the set $K \cup L$ of $C_{i-1} \cup C_{i}$ becomes, after the identifications made in defining $D$, an open set of $D$ containing $u \times v$. The collection of all such sets constitutes the covering $\mathfrak{Y}$. Since each $\mathfrak{B}_{i}$ is a star-refinement of $\mathfrak{B}_{i-1}$, it is easy to see that $\zeta^{-1}(\mathfrak{Y})$ is a refinement of $\mathfrak{X}$ and hence that $f^{-1}(\mathfrak{Y})$ is a refinement of $\mathfrak{B}$. 
It is now easy to finish the proof of the theorem. First we make a barycentric subdivision of $D$, thus obtaining a simplicial complex $E$. Let $e_{1}$ be a vertex of $E$, and let $\bar{S}_{i}$ have the same meaning for $E$ as $S_{i}$ has for $N(\mathfrak{U})$ above. Next we subdivide $\bar{S}_{2}$ simplicially until the star of each vertex in the induced subdivision of $\bar{S}_{1}$ is contained in some element of $\mathfrak{Y}$. Then we subdivide $\bar{S}_{3}$ simplicially, without introducing any new vertices in $\bar{S}_{1}$, until each vertex of the induced subdivision of $\bar{S}_{2}$ has its star contained in some element of $\mathfrak{Y}$.

Continuing in this fashion, all of $E$ is subdivided in such a way that each cell of $D$ is divided into a finite number of simplexes.

Now let $\$$ be the covering of $D$ by the stars of the vertices of the subdivision of $E$. By construction, $\mathbb{B}$ is a refinement of $\mathfrak{Y}$. Since each cell of $D$ is of dimension at most $n+m$, the same is true of $E$ and of its subdivision. Hence, order $B \leqq n+m+1$. Clearly $B$ is star-finite. Hence $f^{-1}(\Omega)$ is a star-finite covering of $A \times B$, of order not greater than $n+m+1$, and a refinement of $\mathfrak{W}_{0}$, which proves the theorem.

To show that the product of two $S$-spaces need not be an $S$-space, we appeal to an example, constructed by Sorgenfrey [4], of a paracompact space whose product with itself is not paracompact. It is only necessary to observe that this space is actually an $S$-space, as is easily seen by an inspection of his proof.

Finally, we remark that Hemmingsen [3] has shown that the dimension theorem holds for the product of two compact spaces, and Dieudonné [1] has shown that the product of a compact space and a paracompact space is paracompact. Thus, the only unsettled question in this direction is that concerning the dimension of the product of a compact and paracompact space. It is clear that the method used above cannot be used in this case.

\section{BIBLIOGRAPHY}

1. J. Dieudonné, Une gênêralization des espaces compacts, J. Math. Pures Appl. (9) vol. 23 (1944) pp. 65-76.

2. C. H. Dowker, Mapping theorems for non-compact spaces, Amer. J. Math. vol. 69 (1947) pp. 200-242.

3. E. Hemmingsen, Some theorems in dimension theory for normal spaces, Duke Math. J. vol. 13 (1946) pp. 495-504.

4. R. H. Sorgenfrey, On the topological product of paracompact spaces, Bull. Amer. Math. Soc. vol. 53 (1947) pp. 631-632.

YALE UNIVERSITY 